BMJ Paediatrics Open

\title{
Immunoglobulin for Kawasaki disease: a 3-year retrospective audit
}

\author{
Beth Pascall, ${ }^{\oplus 1}$ Arjuna Thakker, ${ }^{\oplus}$ Ying Foo, ${ }^{1}$ Pradip Thakker ${ }^{1}$
}

To cite: Pascall B, Thakker A, Foo $Y$, et al. Immunoglobulin for Kawasaki disease: a 3-year retrospective audit. BMJ Paediatrics Open 2019;3:e000451. doi:10.1136/ bmjpo-2019-000451

- Additional material is published online only. To view please visit the journal online (http://dx.doi.org/10.1136/ bmjpo-2019-000451).

Received 5 April 2019 Revised 27 May 2019 Accepted 1 June 2019
Check for updates

(C) Author(s) (or their employer(s)) 2019. Re-use permitted under CC BY-NC. No commercial re-use. See rights and permissions. Published by BMJ.

${ }^{1}$ Paediatrics, Nottingham University Hospitals NHS Trust Nottingham, UK

${ }^{2}$ University of Birmingham College of Medical and Dental Sciences, Birmingham, UK

Correspondence to Dr Beth Pascall; elizabeth. pascall@nhs.net

\section{ABSTRACT}

Aim To evaluate whether intravenous immunoglobulin (IVIG) use in children with suspected Kawasaki disease (KD) was given according to local trust and the newly revised American Heart Association (AHA) guidelines.

Methods In our tertiary hospital, any child with suspected KD given IVIG, over the past 3 years, was identified. Their electronic notes were then reviewed. Results Ten patients were identified. Nine patients had a fever lasting 5 days or more. Four patients had either $5 / 5$ or $4 / 5$ of the diagnostic criteria for KD and were diagnosed with complete KD. The remaining six patients were suspected to have incomplete KD. 7/10 patients received IVIG within 10 days of onset of illness. Patients suspected to have incomplete KD experienced a mean delay in administration of IVIG of 5.3 days compared with those with complete KD. In four patients, an alternative diagnosis was established. Three patients had coronary artery abnormalities on first echocardiogram. From these patients, only one had a follow-up echocardiogram recorded in their notes. No patient had more than one follow-up echocardiogram (at both 2 and 6 weeks). Conclusion Identifying patients with incomplete $\mathrm{KD}$ is more difficult than identifying those with complete $\mathrm{KD}$ and any delay in giving IVIG could be due to this reason. This audit suggests that increasing awareness of incomplete $\mathrm{KD}$ and a clear guideline will aid prompter diagnosis and administration of IVIG. This audit also suggests that all patients with KD should receive more than one follow-up echocardiogram.

\section{INTRODUCTION}

Kawasaki disease (KD) is a vasculitis affecting small-sized and medium-sized arteries, predominantly affecting children under 5 years. It is characterised by a prolonged fever lasting 5 days or more and coronary artery aneurysms if left untreated. ${ }^{1}$ Timely administration of intravenous immunoglobulin (IVIG) has been shown to reduce the incidence of coronary artery aneurysms from anywhere between $5 \%$ and $26 \%{ }^{2}$

The diagnostic criteria for $\mathrm{KD}$ must include a fever lasting 5 days or more, followed by oral mucosal changes, bilateral non-exudative conjunctivitis, a polymorphous rash, cervical lymphadenopathy, erythema and oedema followed by desquamation of the extremities. Classic or 'complete KD' can be diagnosed with a fever lasting a minimum of 4 days and

\section{What is known about this subject?}

Failure to recognise and treat Kawasaki disease promptly is associated with a higher risk of development of coronary artery aneurysms.

- Patients with incomplete Kawasaki disease (fever with fewer than four diagnostic features) may still develop coronary artery aneurysms.

\section{What this study adds?}

Patients with incomplete Kawasaki disease (KD) may experience a delay in intravenous immunoglobulin administration compared with those with complete KD.

- The algorithm for incomplete KD should be considered when incomplete KD is suspected in any patient. In any uncertainty, a KD expert should be consulted.

- Guidelines must emphasise the importance of follow-up echocardiograms in all patients diagnosed with KD.

at least four out of the five mentioned clinical features. However, as this criterion cannot identify all children with $\mathrm{KD}$, the diagnosis of 'incomplete KD' should be considered in children with prolonged unexplained fever associated with any of the principle clinical features of the disease. Incomplete $\mathrm{KD}$ is a challenging diagnosis to make, especially as fever is a very common presentation of children to hospital. Those under 6 months of age without conjunctivitis and mucosal changes are at particular risk of delayed diagnosis. $15 \%$ to $20 \%$ of patients who do not meet the criteria for complete $\mathrm{KD}$ still develop aneurysms, ${ }^{4}$ and therefore urgent treatment and echocardiogram monitoring remain vital in this group.

Where incomplete $\mathrm{KD}$ is being considered, both AHA and local trust guidelines recommend using a diagnostic algorithm for treatment decisions (online supplementary figures 1 and 2). The algorithm used in the local trust guideline closely models the algorithm used in the gold standard AHA guidelines (there 
remain subtle differences). Despite the use of algorithms, IVIG prescription and administration is closely controlled within the trust and the decision to administer IVIG is taken by a consultant, whose decision is then screened by a panel of 'experts' including a microbiologist and senior pharmacist.

In May 2016, a National Health Service Patient Safety Alert was published to increase awareness of the "risk of death and serious harm from failure to recognise acute coronary syndromes in patients with $\mathrm{KD}^{\prime} .5$ This was closely followed with the release of the American Heart Association (AHA) revised guideline ${ }^{6}$ in April 2017, providing updated recommendations on the diagnosis, treatment and long-term management of $\mathrm{KD}$. In view of both the updated guidelines and a national pledge for early diagnosis of the disease, a 3-year audit of $\mathrm{KD}$ cases was performed, focusing on length of time from presentation to diagnosis and treatment.

\section{METHODS}

The audit was approved by the local trust. A list was obtained from the hospital pharmacy of all paediatric patients prescribed IVIG for KD from May 2014 to May 2017. Electronic notes were looked at by the authors and the following factors were reviewed: age at diagnosis, sex, ethnicity, weight, which diagnostic clinical features present, complete or incomplete $\mathrm{KD}$ diagnosis made, if incomplete, whether the AHA or the local trust guidelines was followed, duration of fever, fever peak, antibiotics given, length of hospital stay, laboratory investigations, time from onset of illness to IVIG, time from diagnosis/consideration of diagnosis to IVIG, whether correct dose of IVIG was given, time from IVIG to termination of fever, whether two doses IVIG or adjunct therapies were given, whether initial and maintenance aspirin doses given, time from diagnosis/consideration of diagnosis to first echocardiogram, timing of subsequent echocardiograms, length of follow-up and whether an alternative diagnosis was eventually established.

\section{Patient involvement}

Patients were not directly involved in the design of this study.

\section{RESULTS}

Eleven patients were identified from our 3-year retrospective search. One patient was excluded due to transfer to a cardiac centre following IVIG administration, meaning electronic notes were not available. 7 out of 10 patients were male. The mean age of the patients was 32 months with the youngest patient being 8 weeks and the oldest patient being 5 years of age. As some patients were transferred from other hospitals, the combined total hospital stay ranged from 3 to 14 days, with a mean of 7 days in hospit

9 patients out of 10 were found to have a fever lasting 5 days or more. Only one patient had a fever lasting less than 5 days. The average length of time a patient had a fever was approximately 8 days. Five patients had a highest recorded temperature of more than $39^{\circ} \mathrm{C}$.

Two patients satisfied all six diagnostic criteria for classic KD (fever+five clinical signs) set out in the AHA and trust guidelines. Two patients satisfied five, two satisfied four, two satisfied two and two satisfied one (table 1). The most common clinical feature, aside from fever, was extremity changes, present in seven patients. The least common clinical feature was cervical lymphadenopathy, present in three patients.

Four patients were diagnosed with complete $\mathrm{KD}$ at the time (table 2), meeting the diagnostic criteria of prolonged fever and at least four out of the five clinical signs needed to make the diagnosis. The remaining six patients were suspected to have incomplete $\mathrm{KD}$ at the time, with five out of six patients presenting with prolonged fever but fewer than four principal clinical signs (table 1).

Out of the six patients with suspected incomplete KD, only one satisfied both the AHA and trust algorithm. This was due to a positive echocardiogram result. The remaining five patients did not satisfy either algorithms due to a strict cut-off for laboratory findings (online supplementary figures 1 and 2).

An alternative diagnosis was eventually established in four patients (table 2). From these patients, all four were suspected to have incomplete $\mathrm{KD}$ at the time of presentation. These patients were prescribed IVIG even though they did not meet both the AHA and trust algorithm for incomplete $\mathrm{KD}$.

\begin{tabular}{lllllllllll}
\hline Table 1 & Clinical criteria for Kawasaki disease and patient population checklist for criteria met \\
\hline Patient & $\mathbf{1}$ & $\mathbf{2}$ & $\mathbf{3}$ & $\mathbf{4}$ & $\mathbf{5}$ & $\mathbf{6}$ & $\mathbf{7}$ & $\mathbf{8}$ & $\mathbf{9}$ & $\mathbf{1 0}$ \\
\hline$\geq 5$ days of fever & Yes & Yes & Yes & Yes & No & Yes & Yes & Yes & Yes & Yes \\
Mucous membrane involvement & Yes & Yes & No & Yes & Yes & No & No & No & No & Yes \\
Bilateral non-exudative conjunctivitis & Yes & Yes & No & Yes & Yes & No & No & No & No & Yes \\
Polymorphous erythematous rash & Yes & Yes & Yes & Yes & No & No & Yes & No & No & Yes \\
Extremity changes & Yes & Yes & Yes & Yes & No & No & Yes & No & Yes & Yes \\
Cervical lymphadenopthy $>1.5 \mathrm{~cm}$ & No & No & Yes & Yes & No & No & Yes & No & No & Yes \\
\hline
\end{tabular}


Table 2 Summary table for patient population

\begin{tabular}{clllll}
\hline & $\begin{array}{l}\text { Suspected } \\
\text { diagnosis at the } \\
\text { time (complete } \\
\text { or incomplete } \\
\text { Kawasaki) }\end{array}$ & $\begin{array}{l}\text { If incomplete, was } \\
\text { AHA algorithm } \\
\text { criteria met? }\end{array}$ & $\begin{array}{l}\text { If incomplete, was } \\
\text { trust algorithm } \\
\text { criteria met? }\end{array}$ & $\begin{array}{l}\text { Alternative diagnosis } \\
\text { eventually established? }\end{array}$ & $\begin{array}{l}\text { Time to IVIG from } \\
\text { onset of illness } \\
\text { (days) }\end{array}$ \\
\hline 1 & Complete & NA & NA & No & 6 \\
\hline 2 & Complete & NA & NA & No & 8 \\
\hline 3 & Incomplete & Yes & Yes & No & 10 \\
\hline 4 & Complete & NA & NA & No & Rheumatological diagnosis \\
\hline 5 & Incomplete & No & No & Stills disease & Many \\
\hline 7 & Incomplete & No & No & Haemophilus and rhinovirus \\
pneumonia with effusion & 24 \\
\hline 9 & Incomplete & No & No & Polyarteritis nodosa & 6 \\
\hline 10 & Incomplete & No & No & No & No \\
\hline
\end{tabular}

The time taken from the onset of the initial illness to IVIG ranged from 5 to 24 days, with seven patients given IVIG within 10 days of illness. One patient received IVIG after 24 days due to a peeling rash and raised erythrocyte sedimentation rate (ESR). The other two patients received IVIG after 10 days either due to a raised C-reactive protein (CRP) or a raised ESR. The time taken from the initial diagnosis/consideration of $\mathrm{KD}$ to giving IVIG ranged from less than 24 hours to up to 4 days. One patient received a second dose of IVIG. Cases of incomplete $\mathrm{KD}$ experienced a mean delay in administration of IVIG of 5.3 days compared with those diagnosed with complete KD (online supplementary figure 1 ).

Seven patients received aspirin at $50 \mathrm{mg} / \mathrm{kg}$ until their fever resolved. One patient was also treated with a combination of steroids and infliximab due to an ongoing fever and worsening left coronary artery dilation.

The mean time from diagnosis/consideration of KD to first echocardiogram was 50 hours, with the longest time being 96 hours for one patient. The first echocardiogram showed coronary artery abnormalities in three patients.
After 1-2 weeks following the first echocardiogram, three patients had a repeat echocardiogram. Five patients had a repeat echocardiogram after 4-6weeks following the first echocardiogram.

Four patients still have active ongoing follow-up and two have been transferred to another centre for ongoing care.

\section{DISCUSSION}

Despite evidence showing that the incidence of KD is increasing, ${ }^{7}$ it still remains a rare condition in the UK, with an incidence of 9.1 per 100000 in children under 5 years. ${ }^{8}$ In our hospital, an average of 3.3 patients per year were treated for suspected KD, with a mean age of 32 months and a typical age distribution.

A summary of the audit standards and outcomes is shown in table 3. Fever for more than 5 days was the most common clinical feature and was present in nine patients. As fever is a common reason for paediatric presentation to hospital, this makes a diagnosis of $\mathrm{KD}$

Table 3 Summary of standards set out by the 2017 American Heart Association guideline 'diagnosis, treatment and longterm management of KD' and the outcomes of the audit

\begin{tabular}{lll}
\hline Audit criteria & Target & Exceptions \\
\hline $\begin{array}{l}\text { The diagnosis of KD should be made based on the listed } \\
\text { diagnostic criteria or according to the AHA algorithm in cases } \\
\text { of incomplete KD }\end{array}$ & $100 \%$ & $\begin{array}{l}\text { This incomplete KD algorithm is not evidence } \\
\text { based but represents the opinion of an expert } \\
\text { committee; therefore, exceptions may arise } \\
\text { following consultation with an expert }\end{array}$ \\
$\begin{array}{l}\text { Echocardiography should be performed when the diagnosis } \\
\text { of KD is considered, and repeated within 1-2 weeks and 4-6 } \\
\text { weeks in uncomplicated patients }\end{array}$ & $100 \%$ & None \\
$\begin{array}{l}\text { Patients with complete or incomplete KD should be treated } \\
\text { with high dose IVIG (2 mg/kg) within } 10 \text { days of illness onset }\end{array}$ & $100 \%$ & $\begin{array}{l}\text { If delayed presentation to hospital, IVIG should } \\
\text { be given as soon as possible after diagnosis }\end{array}$ \\
\hline $\begin{array}{l}\text { A second dose of IVIG should only be given to patients with } \\
\text { persistent fever } 36 \text { hours after the initial dose }\end{array}$ & $100 \%$ & \begin{tabular}{l} 
None \\
\hline
\end{tabular} \\
\hline
\end{tabular}

IVIG, intravenous immunoglobulin; KD, Kawasaki disease. 
extremely challenging. The least common clinical feature was cervical lymphadenopathy, which correlates with the literature findings of lymphadenopathy being present only in $52 \%$ of patients with $\mathrm{KD} .{ }^{9}$ Patients presenting with both fever and cervical lymphadenopathy can often be misdiagnosed as having bacterial lymphadenitis. In our cohort, four patients were presented with fever and cervical lymphadenopathy. From these patients, two had definitive complete KD and two were suspected to have incomplete KD. It is therefore important to consider $\mathrm{KD}$ in any patient suspected of having bacterial lymphadenitis, especially in incomplete cases where clinical features of KD may be absent. ${ }^{10}$

All patients received the correct dose of IVIG. High dose IVIG $(2 \mathrm{~g} / \mathrm{kg})$ has been conclusively shown to be more effective than other dose regimens used in the past, and as a result this dose is used consistently across the UK and USA, minimising potential for dosing errors. The AHA guideline states that IVIG should be given within 10 days of illness onset (ideally as soon as possible after diagnosis). We achieved this in seven of 10 patients. The reason for the delay in the remaining three patients was possibly due to uncertainty regarding the diagnosis. According to the AHA guidelines, it is reasonable to give IVIG after the 10th day of illness if the child has persistent fever or coronary artery abnormalities together with ongoing systemic inflammation manifested by elevated CRP or ESR. This was likely to be the case in all three patients who received IVIG after the 10th day of illness, despite only one of them having a documented reason in their electronic notes (online supplementary table 1 ).

Our data clearly showed that those patients who fulfilled the diagnostic criteria for complete KD received IVIG earlier than those with incomplete KD. It is well recognised that patients with incomplete $\mathrm{KD}$ who have prolonged fever but fewer than four of the diagnostic clinical features may still develop cardiovascular complications. As was the case with one patient, who developed coronary artery dilation. Despite this happening to only one patient from six, this fits with the predicted incidence of cardiovascular complications in those with incomplete KD. ${ }^{11}$ Therefore, IVIG administration in incomplete KD cases remains as clinically urgent as for complete $\mathrm{KD}$ cases.

Incomplete $\mathrm{KD}$ is more common in infants under 6 months $^{4}$; however, in our group of patients, the mean age for those with presumed incomplete $\mathrm{KD}$ was approximately 2.5 years, with no patients under 6 months of age. An alternative diagnosis was eventually established in four of the six patients, which was not surprising, given that neither the AHA nor local trust algorithm was met in these patients. This also supports the robustness of the AHA and local trust algorithms and suggests we should be more stringent with its use.

Despite the similarity between the AHA and local trust algorithms, the AHA guidelines further state that in any circumstance of uncertainty, a 'KD expert' should be consulted. The local guidelines, however, recommend referring to the consultant responsible for the patient. In most cases, this is likely to be paediatrician, and while most paediatricians are knowledgeable about $\mathrm{KD}$, they are not KD specialists per se. Therefore, experts in KD should be preferably consulted in any uncertain cases of KD.

Persistence or recurrence of fever at least 36 hours after the initial IVIG infusion occurs in approximately $11 \%$ of patients with $\mathrm{KD}^{12}$ and is termed IVIG resistance. The AHA guidelines recommend considering a second dose of IVIG after at least 36 hours in the case of IVIG resistance, although there is no strong evidence to support this. One of our patients received this second dose, which appeared to be in accordance with the AHA recommendations. One patient went on to receive methylprednisolone and infliximab due to ongoing fever and worsening coronary dilation. This was also appropriate and consistent with the AHA recommendations.

The AHA guidelines also state that echocardiograms should be performed at first presentation, 1-2 weeks and at 4-6 weeks as a minimum in uncomplicated patients. We did not achieve these follow-up goals in any of the 10 patients. All patients had an echocardiogram at presentation but either the 1-2 week or 4-6 week echocardiogram was missed, despite these targets also being clearly stated in the trust guideline. As of yet, there are no clinical repercussions from this, but further investigation is required to determine why this happened. Similarly, despite incomplete data, the serial echocardiograms are recommended by AHA in those patients with an abnormal initial echocardiogram; however, this was not adhered to. The AHA guidelines recommend at least twice weekly echocardiograms in these patients until luminal dimensions have stopped progressing, then weekly in the first 45 days of illness, then monthly until the third month after onset. The reason for this not being met could be that these standards are simply unrealistic given the limited resources in the UK. A detailed assessment of echocardiogram techniques and reporting, including the use of Z-scores to assess luminal dimensions of coronary arteries, was beyond the scope of this audit. However, it a potential area for future audit as clear guidelines are set out by the AHA.

\section{Recommendations}

The most striking finding was the delay in administration of IVIG in patients with incomplete KD. This was most likely due to the difficulty in identifying patients with incomplete $\mathrm{KD}$, as well as unawareness of the local trust algorithm. Increased awareness of this algorithm is therefore necessary, and the AHA algorithm should be used in the local guideline. Further, patients who do not meet the criteria set out in the algorithm, should not receive IVIG, unless there is either a clear alternative indication or a $\mathrm{KD}$ expert is consulted.

Second, all uncomplicated patients only received one follow-up echocardiogram, while complicated patients were not followed up closely enough. Although the 
reason for this requires further research, a strategy needs to be put in place to ensure this does not happen. This could mean specifying that all follow-up echocardiograms need to be booked by the responsible clinician before discharge.

The local Kawasaki guideline should be updated to reflect the above recommendations, followed by reaudit in $12-24$ months.

Contributors PT presented the idea of this audit and supervised the process. BP, $\mathrm{FY}$ and AT contributed to collecting the data and writing the manuscript.

Funding The authors have not declared a specific grant for this research from any funding agency in the public, commercial or not-for-profit sectors.

Competing interests None declared.

Patient consent for publication Not required.

Data availability statement All data relevant to the study are included in the article or uploaded as supplementary information.

Open access This is an open access article distributed in accordance with the Creative Commons Attribution Non Commercial (CC BY-NC 4.0) license, which permits others to distribute, remix, adapt, build upon this work non-commercially, and license their derivative works on different terms, provided the original work is properly cited, appropriate credit is given, any changes made indicated, and the use is non-commercial. See: http://creativecommons.org/licenses/by-nc/4.0/.

\section{REFERENCES}

1. McCrindle BW, Rowley AH, Newburger JW, et al. Diagnosis, treatment, and long-term management of Kawasaki disease: a scientific statement for health professionals from the American heart association. Circulation 2017;135.
2. Terai M, Shulman ST, Minich LL. Prevalence of coronary artery abnormalities in Kawasaki disease is highly dependent on gamma globulin dose but independent of salicylate dose. $J$ Pediatr 1997;131:888-93.

3. Sleeper LA, Atz AM, McCrindle BW, et al. Delayed diagnosis of Kawasaki disease: what are the risk factors? Pediatrics 2007;120:e1434-40.

4. Rowley AH. Incomplete (atypical) Kawasaki disease. Pediatr Infect Dis J 2002;21:563-5.

5. NHS Improvement. Patient Safety Alert: Risk of death and serious harm from failure to recognise acute coronary syndromes in Kawasaki disease patients [Internet], 2016. Available: https:// improvement.nhs.uk/documents/92/Patient_Safety_Alert_Stage_1 Failure_to_recognise_coronary_syndromes_in_Kawasa_ivbyZC0.pdf [Accessed 14 Jun 2018].

6. McCrindle BW, Rowley AH, Newburger JW, et al. Diagnosis, treatment, and long-term management of Kawasaki disease: a scientific statement for health professionals from the American heart association. Circulation 2017;135:e927-99.

7. Harnden A, Alves B, Sheikh A. Rising incidence of Kawasaki disease in England: analysis of hospital admission data. BMJ 2002;324:1424-5.

8. Hall GC, Tulloh LE, Tulloh RMR. Kawasaki disease incidence in children and adolescents: an observational study in primary care. $\mathrm{Br}$ $J$ Gen Pract 2016;66:e271-6.

9. April MM, Burns JC, Newburger JW, et al. Kawasaki disease and cervical adenopathy. Arch Otolaryngol Head Neck Surg 1989;115:512-4.

10. Kubota M, Usami I, Yamakawa M, et al. Kawasaki disease with lymphadenopathy and fever as sole initial manifestations. J Paediatr Child Health 2008;44:359-62.

11. Duarte R, Cisneros S, Fernandez G, et al. Kawasaki disease: a review with emphasis on cardiovascular complications. Insights Imaging 2010;1:223-31.

12. Durongpisitkul K, Soongswang J, Laohaprasitiporn D, et al. Immunoglobulin failure and retreatment in Kawasaki disease. Pediatr Cardiol 2003;24:145-8. 